\title{
Factores que influyen en la supervivencia de la fístula arteriovenosa interna y su relación con la técnica de punción
}

\author{
Aida Delgado Ramírez, Estíbaliz Ruiz García, Luis Ignacio Latorre López, Rodolfo Crespo Montero \\ Facultad de Medicina y Enfermería. Universidad de Córdoba. España
}

\section{Resumen}

Introducción: La siguiente revisión bibliográfica se realiza con el propósito de recopilar las causas de fracaso de la fístula arteriovenosa interna, haciendo especial hincapié en la relación entre la técnica de punción y fracaso de la misma, dada la importancia que el manejo y cuidados de enfermería tienen en el acceso vascular del paciente en hemodiálisis.

Objetivo: Revisar la producción científica disponible sobre fallo primario o secundario de la fístula arteriovenosa interna.

Metodología: Se realizó una revisión sistemática no metaanalítica, con búsqueda de información en las bases de datos Medline, Proquest, Scopus y Google Scholar. Se incluyeron artículos en español, inglés y francés.

Resultados: Se revisaron 38 artículos publicados entre el año 2001 y 2016. La mayoría de ellos estudios observacionales. Tras el análisis de todas las variables se encontró que la edad avanzada, el género femenino, estado inflamatorio y de coagulación, la diabetes, episodios de hipotensión, localización y uso de catéter venoso central influyen negativamente en la supervivencia de la fístula arteriovenosa interna. En cuanto a la influencia de las diferentes técnicas de punción de la fístula, en la supervivencia de la misma, no hay datos concluyentes a favor de la ninguna técnica.

Conclusiones: Podemos concluir que los factores que influyen negativamente en la supervivencia de la fístula arteriovenosa interna son: edad avanzada, género

Correspondencia:

Aida Delgado Ramírez

C/ Hermanos Álvarez Quintero, $\mathrm{n}^{\circ} 2$

Montilla, 14550 Córdoba

E-mail: aida_dr_94@hotmail.com femenino, diabetes, estado inflamatorio, episodios de hipotensión, localización distal y uso previo de catéter venoso central.

PALABRAS CLAVE

- FISTULA ARTERIOVENOSA

- TROMBOSIS

- HEMODIÁLISIS

Factors which influence the survival of arteriovenous fistula and their relationship to the puncture technique

\section{Abstract}

Introduction: The following literature review aims to collect the causes of failure of arteriovenous fistula, with particular emphasis on the relationship between puncture technique and the failure of it, given the importance of management and nursing care in the vascular access of hemodialysis patients.

Objective: To review the available scientific literature about the primary or secondary failure of arteriovenous fistula.

Methods: A, non-meta-analytic, systematic review was carried out with information search in the following databases: Medline, ProQuest, Scopus and Google Scholar. Articles in Spanish, English and French are included.

Results: The review includes 38 articles published between 2001 and 2016. Most of them are observational studies. After analyzing all the variables, we found that older age, female gender, inflammatory and 
coagulation status, diabetes, episodes of hypotension, location and use of central venous catheter adversely affect the survival of arteriovenous fistula. Regarding the influence of different needling techniques in the survival of the fistula, there is no conclusive evidence in favor of any technique.

Conclusions: We can conclude that factors which adversely affect the survival of arteriovenous fistula are: advanced age, female gender, diabetes, inflammatory state, episodes of hypotension, distal location and previous use of central venous catheter.

\section{KEYWORDS}

\section{- ARTERIOVENOUS FISTULA \\ - THROMBOSIS \\ - HEMODIALYSIS}

\section{Introducción}

La prevalencia de la Enfermedad Renal Crónica Avanzada (ERCA) enfermedad va en aumento y se trata de un problema cada vez con mayor repercusión en todo el mundo, y se estima que en España padecen ERCA unos 4 millones de personas ${ }^{1,2}$. De ellas, unas 55.156 están en tratamiento renal sustitutivo (TRS), de los que el $51^{\prime} 9 \%$ están con un trasplante renal funcionante, un $5^{\prime} 5 \%$ en diálisis peritoneal y un $42^{\prime} 6 \%$ en $\mathrm{HD}^{3}$.

Esta situación es muy particular de nuestro país, por el alto porcentaje de donación de órganos que se obtie$n e^{4}$. No ocurre lo mismo en el resto del mundo, en el que el tratamiento de elección es la HD5. La HD es un TRS que precisa de un circuito sanguíneo extracorpóreo, un monitor específico, y sobre todo, que el paciente tenga un acceso vascular (AV) para poder depurar su sangre. El AV se trata del factor más importante que determina el éxito o fracaso de los programas de HD crónica; y además el fallo del mismo, es la primera causa por la que los enfermos en HD suelen ingresar en el hospital ${ }^{6,7}$.

Existen 3 tipos de AV para HD: el catéter venoso central (CVC), el injerto, habitualmente de politetrafluoroetileno expandido (PTFE), y la fístula arteriovenosa interna (FAVI). De estos, el de elección es la FAVI, que ha demostrado ser el AV que mejor cumple tres requisitos fundamentales: permitir el abordaje seguro y continuado del sistema vascular con una buena permeabilidad, proporcionar flujos suficientes para realizar HD de forma adecuada con la dosis correcta, y carecer de complicaciones o ser la que menos tiene ${ }^{8,9}$.

La FAVI es la unión directa entre una arteria y una vena subyacente, creada quirúrgicamente. Generalmente, se construyen en la extremidad superior, de preferencia en el brazo no dominante y en la localización más distal posible, para preservar las proximales en caso de que la primera FAVI fracase, aunque pueden construirse en cualquier parte del cuerpo, las más frecuentes son la radio-cefálica de Cimino-Brescia y la braquiocefálica ${ }^{10}$.

A pesar de ser la FAVI el AV más seguro, no está exenta de complicaciones y su duración es limitada. Una parte de los fallos se producen en el proceso de maduración de la misma, es decir, desde su creación hasta la primera punción, conocido como fallo primario o de maduración, y una vez se ha conseguido una FAVI funcionante, esto es, tras las primeras punciones, el fallo se denomina secundario, y suele ocurrir principalmente debido a estenosis del acceso, que habitualmente progresa hacia una trombosis o fracaso de la misma $^{11,12}$. Existen además, otras complicaciones no trombóticas como son: la formación de aneurismas y pseudo-aneurismas, la isquemia, el síndrome del robo, la hemorragia y la rotura del acceso, las infecciones y otras complicaciones como la isquemia distal y la hipertensión venosa ${ }^{13}$.

Por tanto, es imprescindible preservar y evitar el fracaso de la FAVI a toda costa, mediante vigilancia y una adecuada técnica en su manipulación, ya que en el que caso de que el fracaso se produzca, al paciente se le tiene que colocar un acceso temporal, que suele ser un CVC, mientras se le realiza la cirugía para crear otra FAVI o en el caso de que no fuera posible, un injerto de PTFE, necesitando en ocasiones, ingreso hospitalario con el consiguiente estrés para el mismo, originando además un aumento de los costes ${ }^{14,15}$. Además, durante este periodo la morbimortalidad del paciente aumenta exponencialmente debido a la alta tasa de complicaciones que ocasiona un $\mathrm{CVC}^{16}$.

Por todo ello, nos planteamos la presente revisión bibliográfica, con el propósito de determinar las causas de fallo de la FAVI, haciendo especial hincapié en la relación entre la técnica de punción y fracaso de la misma. 


\section{Objetivos}

Revisar la bibliografía existente sobre el fallo primario o secundario de la FAVI en pacientes en HD.

Objetivos específicos:

1. Determinar los factores de riesgo más influyentes en el fracaso de la FAVI.

2. Analizar la relación entre técnicas de punción y fallo de la FAVI.

\section{Metodología}

El diseño empleado para el presente estudio ha sido el de revisión bibliográfica, para la cual se ha llevado a cabo una búsqueda en diversas bases de datos, con el objetivo de obtener la mayor información posible sobre el tema a tratar. Las bases de datos consultadas han sido: Medline, Proquest, Scopus y Google Scholar, en el tiempo comprendido entre el 15 de febrero y el 30 de abril de 2016.

La estrategia de búsqueda en las bases de datos, fue mediante las palabras clave: supervivencia fístula arteriovenosa ("arteriovenous fistula survival"), hemodiálisis ("hemodialysis"), trombosis ("thrombosis"), fallo fístula arteriovenosa ("arteriovenous fistula failure"), y canulación ("cannulation"). En las bases de datos que tenían la opción de tesauros fueron utilizados los siguientes: "arteriovenous fistula", "thrombosis", "hemodialysis". Relacionando estos términos con los operadores boleanos "and", "or" y "not", según conveniencia.

Los criterios de selección fueron que los artículos incluyeran los descriptores en título, resumen o palabras clave, y que hubieran sido publicados en los últimos 15 años. En las bases de datos que lo permitían se limitaron las áreas temáticas a aquellas relacionadas con ciencias de la salud, y se limitó la búsqueda a aquellos artículos que tuvieran la disponibilidad de texto completo.

Los criterios de inclusión fueron:

- Artículos que analizaran las causas del fracaso de la FAVI.

- Artículos que trataran sobre técnicas de punción y fracaso y/o complicaciones de la FAVI.

- Artículos sobre vigilancia y monitorización de la FAVI.

- Artículos originales completos.

- Artículos de revisión.
Los criterios de exclusión fueron:

- Artículos que no presentaran resultados concluyentes con respecto al tema del estudio.

En un primer momento se encontraron 118 artículos, seleccionados únicamente mediante la lectura del título y el resumen; más tarde, se descartaron aquellos que mediante una lectura del texto completo se decidió que no tenían relación o no ofrecían resultados de utilidad para el tema a estudiar; finalmente, fueron seleccionados aquellos estudios que mediante una lectura exhaustiva se determinó que tenían realmente validez para nuestro estudio y desechamos aquellos que no encajaban por completo con el tema. Al recopilar todos los artículos importantes sobre este tema, se realizó una búsqueda del factor de impacto de las revistas de las que son extraídos, para así poder clasificarlos según el factor de impacto de la misma, determinado por cuartiles Q1, Q2, Q3 y Q4, tanto en Journal Citation Report (JCR) como en SCimago Journal Rank (SJR).

Posteriormente, se analizó la calidad metodológica de los artículos seleccionados para la investigación según los criterios adaptados de Berra, Elorza-Ricart, Estrada y Sánchez, $2008^{17}$, de los cuales solamente se encontraron 2 artículos con una calidad baja, 6 con calidad media, y los 30 artículos restantes con una calidad alta.

\section{Resultados}

En total, de los 118 documentos preseleccionados de todas las bases de datos, tras eliminar 56, se quedaron 62 artículos, que después de su lectura completa y tras comprobar que cumplían con los criterios de inclusión/ exclusión establecidos, se seleccionaron 38 , tal como puede observarse en la Tabla 1.

En la Tabla 2, se representan los artículos revisados: autor, año y país de publicación, el tipo de estudio, la muestra estudiada, así como las principales conclusiones.

En la Tabla 3 se muestra se muestra la calidad metodológica de los 38 artículos seleccionados.

Tabla 1. Artículos seleccionados.

\begin{tabular}{lccc}
\hline Base de datos & Titulo + resumen & Texto completo & Selección final \\
\hline Medline & 33 & 21 & 16 \\
\hline ProQuest & 39 & 25 & 11 \\
SCOPUS & 26 & 11 & 8 \\
Google Scholar & 20 & 5 & 3 \\
\hline
\end{tabular}


[ Aida Delgado Ramírez, et al ]

Factores que influyen en la supervivencia de la fístula arteriovenosa interna y su relación con la técnica de punción

Tabla 2. Artículos revisados.

\begin{tabular}{|c|c|c|c|}
\hline Autor, Año, País & Tipo de estudio & Muestra & Principales Resultados \\
\hline $\begin{array}{l}\text { Lazarides MK, Georgiadis } \\
\text { GS, Antoniou GA, et al, 2007, } \\
\text { Grecia }^{18} \\
\text { JCR = Q1 } \\
\text { SJR = Q1 }\end{array}$ & Meta-análisis & $13 * *$ & $\begin{array}{l}\text { La edad avanzada y la FAVI radio } \\
\text { cefálica incrementan el riesgo de } \\
\text { fallo de la misma }\end{array}$ \\
\hline $\begin{array}{l}\text { Cheng Q, Zhao YJ, 2015, } \\
\text { China }^{19} \\
\text { JCR }=\text { Q4 } \\
\text { SJR }=\text { Q3 }\end{array}$ & Estudio observacional & $819 *$ & $\begin{array}{l}\text { La edad avanzada, antecedentes de } \\
\text { hipertensión y diabetes, la presión } \\
\text { arterial baja, el tamaño pequeño } \\
\text { de los vasos, y niveles elevados de } \\
\text { PTH son factores de riesgo para el } \\
\text { fallo de la FAVI }\end{array}$ \\
\hline $\begin{array}{l}\text { Ocak G, Rotmans JI, Vossen } \\
\text { CY, et al, 2013, Holanda }{ }^{11} \\
\text { JCR }=\text { Q3 } \\
\text { SJR = Q2 }\end{array}$ & $\begin{array}{l}\text { Estudio de cohortes } \\
\text { prospectivo multicéntrico }\end{array}$ & $919 *$ & $\begin{array}{l}\text { Enfermedad cardiovascular, uso } \\
\text { previo de catéter venoso central, } \\
\text { niveles bajos de albúmina, y niveles } \\
\text { altos de CRP de alta sensibilidad y } \\
\text { de fetuina-A, están asociados con } \\
\text { el fallo primario de la fistula }\end{array}$ \\
\hline $\begin{array}{l}\text { Chang TI, Paik J, Greene T, } \\
\text { et al, 2011, EEUU } 12 \\
\text { JCR = Q1 } \\
\text { SJR = Q1 }\end{array}$ & $\begin{array}{l}\text { Estudio observacional } \\
\text { multicéntrico retrospectivo }\end{array}$ & $1426 *$ & $\begin{array}{l}\text { Episodios frecuentes de } \\
\text { hipotensión intradialítica y niveles } \\
\text { bajos de presión sanguínea sistólica } \\
\text { pre-diálisis están asociados con } \\
\text { mayores tasas de trombosis de la } \\
\text { FAVI }\end{array}$ \\
\hline $\begin{array}{l}\text { Farber A, Imrey PB, Huber } \\
\text { TS, et al, 2016, EEUU20 } \\
\text { JCR = Q1 } \\
\text { SJR = Q1 }\end{array}$ & $\begin{array}{l}\text { Estudio de cohortes } \\
\text { prospectivo observacional }\end{array}$ & $602^{*}$ & $\begin{array}{l}\text { El género femenino, FAVI en } \\
\text { el antebrazo, tamaño arterial } \\
\text { pequeño, diámetro de la vena } \\
\text { de } 2 \text { a } 3 \mathrm{~mm} \text {, uso de protamina, } \\
\text { "thrill" intraoperatorio ausente o } \\
\text { atenuado, están relacionados con la } \\
\text { trombosis de la FAVI }\end{array}$ \\
\hline $\begin{array}{l}\text { Bashar K, Zafar A, } \\
\text { Elsheikh S, et al, 2015, } \\
\text { Irlanda }{ }^{21} \\
\text { JCR = Q1 } \\
\text { SJR = Q1 }\end{array}$ & $\begin{array}{l}\text { Estudio observacional } \\
\text { retrospective }\end{array}$ & $86 *$ & $\begin{array}{l}\text { El género femenino está } \\
\text { relacionado con el fallo en la } \\
\text { maduración de la FAVI }\end{array}$ \\
\hline $\begin{array}{l}\text { Salmela B, Hartman J, } \\
\text { Peltonen S, et al, 2013, } \\
\text { Finlandia }{ }^{22} \\
\text { JCR }=\text { Q1 } \\
\text { SJR = Q1 }\end{array}$ & Estudio observacional & $219 *$ & $\begin{array}{l}\text { La trombofilia y el género } \\
\text { femenino predisponen a los } \\
\text { pacientes al fallo de la FAVI }\end{array}$ \\
\hline $\begin{array}{l}\text { Masengu A, Maxwell AP, } \\
\text { Hanko JB, 2016, Reino } \\
\text { Unido } \\
\text { SJR = Q3 }\end{array}$ & $\begin{array}{l}\text { Estudio de cohortes } \\
\text { prospectivo }\end{array}$ & $525 *$ & $\begin{array}{l}\text { Género femenino y localización } \\
\text { distal de la FAVI, incrementan el } \\
\text { riesgo de fallo de la misma }\end{array}$ \\
\hline $\begin{array}{l}\text { Tirinescu DC, Bondor CI, } \\
\text { Kacso IM, 2015, Rumania }{ }^{24} \\
\text { SJR = Q3 }\end{array}$ & $\begin{array}{l}\text { Estudio observacional } \\
\text { transversal }\end{array}$ & $97^{*}$ & $\begin{array}{l}\text { Anatomía de los vasos, historia } \\
\text { del acceso vascular (uso previo } \\
\text { de catéter), parámetros del } \\
\text { metabolismo mineral y el género } \\
\text { masculino, son factores de riesgo } \\
\text { para la estenosis de la FAVI }\end{array}$ \\
\hline
\end{tabular}


[ Aida Delgado Ramírez, et al ]

Factores que influyen en la supervivencia de la fístula arteriovenosa interna y su relación con la técnica de punción

\begin{tabular}{|c|c|c|c|}
\hline Autor, Año, País & Tipo de estudio & Muestra & Principales Resultados \\
\hline $\begin{array}{l}\text { Moon J-Y, Lee HM, Lee } \\
\text { S-H, et al, 2015, Korea }{ }^{25} \\
\text { SJR = Q3 }\end{array}$ & $\begin{array}{l}\text { Estudio observacional } \\
\text { prospectivo }\end{array}$ & $478 *$ & $\begin{array}{l}\text { Diabetes, hiperfosfatemia e } \\
\text { hipercalcemia, influyen en la } \\
\text { perdida de permeabilidad primaria } \\
\text { de la FAVI }\end{array}$ \\
\hline $\begin{array}{l}\text { Afsar B, Elsurer R, 2012, } \\
\text { Turquia }{ }^{26} \\
\text { JCR }=\text { Q3 } \\
\text { SJR }=\text { Q3 }\end{array}$ & Estudio retrospectivo & $233 *$ & $\begin{array}{l}\text { El mal control de la glucemia } \\
\text { con niveles altos de hemoglobina } \\
\text { glicosilada se relaciona con el fallo } \\
\text { primario de la FAVI }\end{array}$ \\
\hline $\begin{array}{l}\text { Protack CD, Jain A, Vasilas } \\
\text { P, 2012, EEUU27 } \\
\text { JCR }=Q 1 \\
\text { SJR }=Q 1\end{array}$ & Estudio observacional & $187^{*}$ & $\begin{array}{l}\text { El síndrome metabólico influye en } \\
\text { el fallo de la FAVI }\end{array}$ \\
\hline $\begin{array}{l}\text { Verschuren JJW, 0cak G, } \\
\text { Dekker FW, et al, 2013, } \\
\text { Holanda }{ }^{28} \\
\text { JCR }=\text { Q1 } \\
\text { SJR }=\text { Q1 }\end{array}$ & $\begin{array}{l}\text { Estudio de cohortes } \\
\text { multicéntrico prospectivo }\end{array}$ & $207 *$ & $\begin{array}{l}\text { Los mediadores de la inflamación } \\
\text { (factor V de Leiden y gen LRPI } \\
\text { rs1466535) están asociados con } \\
\text { mayor riesgo de fallo de la FAVI }\end{array}$ \\
\hline $\begin{array}{l}\text { Memetoglu ME, Kehlibar } \\
\text { T, Yilmaz M, et al, 2015, } \\
\text { Turquia }{ }^{29} \\
\text { SJR = Q3 }\end{array}$ & Estudio observacional & $313 *$ & $\begin{array}{l}\text { Mayor heterogeneidad en el } \\
\text { tamaño de los glóbulos rojos y una } \\
\text { actividad inflamatoria aumentada } \\
\text { influyen en el fallo de la FAVI }\end{array}$ \\
\hline $\begin{array}{l}\text { Bilgic A, Ozdemir FN, } \\
\text { Bayraktar N, et al, 2007, } \\
\text { Turquia }{ }^{30} \\
\text { JCR = Q2 } \\
\text { SJR = Q1 }\end{array}$ & Estudio caso control & $82 *$ & $\begin{array}{l}\text { Niveles plasmáticos altos del } \\
\text { receptor de la proteína C } \\
\text { endotelial soluble se relacionan } \\
\text { con la trombosis de la FAVI }\end{array}$ \\
\hline $\begin{array}{l}\text { Hruby Z, Stanek- } \\
\text { Piotrowska M, Turek J, et } \\
\text { al, 2013, Polonia31 } \\
\text { JCR }=\text { Q4 } \\
\text { SJR }=\text { Q3 }\end{array}$ & $\begin{array}{l}\text { Estudio caso control } \\
\text { observacional prospectivo }\end{array}$ & $34 *$ & $\begin{array}{l}\text { La actividad inflamatoria mediada } \\
\text { por los factores de crecimiento de } \\
\text { la pared vascular PDGFßI y TGFß } \\
\text { se relaciona con la alteración de la } \\
\text { función de la FAVI }\end{array}$ \\
\hline $\begin{array}{l}\text { Chang C-J, Ko Y-S, Ko P-J, } \\
\text { et al, 2005, Taiwan } 32 \\
\text { JCR }=\text { Q1 } \\
\text { SJR }=\text { Q1 }\end{array}$ & Estudio observacional & $36 *$ & $\begin{array}{l}\text { La trombosis de la FAVI está } \\
\text { relacionada con una marcada } \\
\text { actividad inflamatoria }\end{array}$ \\
\hline $\begin{array}{l}\text { Brahmbhatt A, Remuzzi A, } \\
\text { Franzoni M, et al, 2016, } \\
\text { EEUU33 } \\
\text { JCR }=Q 1 \\
\text { SJR }=Q 1\end{array}$ & Revisión bibliográfica & No se especifica & $\begin{array}{l}\text { La inflamación, la uremia, la } \\
\text { hipoxia y la tensión tangencial } \\
\text { del endotelio, contribuyen a la } \\
\text { hiperplasia de la íntima y por tanto } \\
\text { a la trombosis de la FAVI }\end{array}$ \\
\hline $\begin{array}{l}\text { Milburn JA, Ford I, Mutch } \\
\text { NJ, et al, 2013, Escocia }{ }^{34} \\
\text { JCR = Q1 } \\
\text { SJR = Q1 }\end{array}$ & $\begin{array}{l}\text { Estudio comparativo } \\
\text { observacional }\end{array}$ & $148 *$ & $\begin{array}{l}\text { Un incremento en los depósitos } \\
\text { de fibrina está relacionado con la } \\
\text { trombosis de la FAVI, en concreto } \\
\text { el aumento del complejo trombina- } \\
\text { antitrombina }\end{array}$ \\
\hline $\begin{array}{l}\text { Bataille S, Burtey S, } \\
\text { Decourt A, et al, 2015, } \\
\text { Francia35 } \\
\text { JCR }=\text { Q4 } \\
\text { SJR }=\text { Q4 }\end{array}$ & Estudio retrospectivo & $192 *$ & $\begin{array}{l}\text { La presencia de anticuerpos } \\
\text { antifosfolípidos, especialmente } \\
\text { el anticoagulante lúpico, está } \\
\text { asociada con la trombosis de la } \\
\text { FAVI }\end{array}$ \\
\hline $\begin{array}{l}\text { Jamshid R, Reza SA, Abbas } \\
\text { G, et al, 2003, Iran }{ }^{36} \\
\text { JCR }=\text { Q3 } \\
\text { SJR }=\text { Q3 }\end{array}$ & $\begin{array}{l}\text { Estudio observacional } \\
\text { prospectivo }\end{array}$ & $230 *$ & $\begin{array}{l}\text { Factores locales como las fistulas } \\
\text { radio-cefálicas y el uso de } \\
\text { tratamiento con EPO son factores } \\
\text { de riesgo para la trombosis de la } \\
\text { FAVI }\end{array}$ \\
\hline
\end{tabular}


[ Aida Delgado Ramírez, et al ]

Factores que influyen en la supervivencia de la fístula arteriovenosa interna y su relación con la técnica de punción

\begin{tabular}{|c|c|c|c|}
\hline Autor, Año, País & Tipo de estudio & Muestra & Principales Resultados \\
\hline $\begin{array}{l}\text { Sánchez Gonzalez, JC, } \\
\text { 2013, España }{ }^{37} \\
\text { SJR = Q3 }\end{array}$ & Estudio caso control & $188^{*}$ & $\begin{array}{l}\text { El uso previo de CVC se asocia con } \\
\text { disfunción de la FAVI }\end{array}$ \\
\hline $\begin{array}{l}\text { Yoo DW, Yoon M, Jun HJ, } \\
2014 \text {, Korea }\end{array}$ & Estudio retrospectivo & $362^{*}$ & $\begin{array}{l}\text { El uso previo de CVC se relaciona } \\
\text { con el fallo de la FAVI }\end{array}$ \\
\hline $\begin{array}{l}\text { Grandaliano G, Teutonico } \\
\text { A, Allegretti A, et al, 2003, } \\
\text { Italia }{ }^{39} \\
\text { JCR = Q1 } \\
\text { SJR = Q1 }\end{array}$ & $\begin{array}{l}\text { Estudio retrospectivo caso } \\
\text { control }\end{array}$ & $87^{*}$ & $\begin{array}{l}\text { El hiperparatiroidismo, hábito } \\
\text { tabáquico, infección por } \\
\text { citomegalovirus, y la dosis de EPO, } \\
\text { incrementan el riesgo de trombosis } \\
\text { de la FAVI }\end{array}$ \\
\hline $\begin{array}{l}\text { Muir CA, Kotwal SS, } \\
\text { Hawley CM, et al, 2014, } \\
\text { Australia }{ }^{40} \\
\text { JCR }=\text { Q1 } \\
\text { SJR = Q1 }\end{array}$ & Estudio observacional & $90 *$ & $\begin{array}{l}\text { La técnica del ojal se asocia con } \\
\text { mayores tasas de infección, y por } \\
\text { tanto de fallo, de la FAVI }\end{array}$ \\
\hline $\begin{array}{l}\text { Parisotto MT, Schoder VU, } \\
\text { Miriunis C, et al, 2014, } \\
\text { Alemania } \\
\text { JCR = Q1 } \\
\text { SJR = Q1 }\end{array}$ & Estudio de cohortes & $7058^{*}$ & $\begin{array}{l}\text { La punción por zonas, la dirección } \\
\text { retrógrada de la aguja arterial } \\
\text { con bisel hacia abajo, el uso de } \\
\text { torniquete o la no aplicación } \\
\text { de presión, y presiones venosas } \\
\text { menores de } 100 \mathrm{~mm} \mathrm{Hg} \mathrm{o} \\
\text { mayores de } 150 \mathrm{~mm} \mathrm{Hg} \text {, se asocian } \\
\text { con mayor riesgo de fallo de la } \\
\text { FAVI }\end{array}$ \\
\hline $\begin{array}{l}\text { Kandil H, Collier S, Yewetu } \\
\text { E, et al, 2014, Reino } \\
\text { Unido } 42 \\
\text { JCR = Q3 } \\
\text { SJR }=\text { Q2 }\end{array}$ & Estudio observacional & $227^{*}$ & $\begin{array}{l}\text { La técnica del ojal aumenta las } \\
\text { tasas de infección de la FAVI, y } \\
\text { dificulta la hemostasia }\end{array}$ \\
\hline $\begin{array}{l}\text { MacRae JM, Ahmed SB, } \\
\text { Atkar R, et al, 2012, } \\
\text { Canada }{ }^{43} \\
\text { JCR }=\text { Q1 } \\
\text { SJR = Q1 }\end{array}$ & $\begin{array}{l}\text { Ensayo controlado } \\
\text { aleatorizado }\end{array}$ & $131^{*}$ & $\begin{array}{l}\text { La técnica del ojal se asocia con } \\
\text { un mayor riesgo de infección de } \\
\text { la FAVI }\end{array}$ \\
\hline $\begin{array}{l}\text { Béchade C, Goovaerts T, } \\
\text { Cougnet } P \text {, et al, 2015, } \\
\text { Belgica }^{44} \\
\text { JCR }=\text { Q1 } \\
\text { SJR = Q1 }\end{array}$ & $\begin{array}{l}\text { Estudio retrospectivo de un } \\
\text { único centro }\end{array}$ & $162^{*}$ & $\begin{array}{l}\text { La técnica del ojal no está } \\
\text { asociada con mayores tasas de } \\
\text { infección }\end{array}$ \\
\hline $\begin{array}{l}\text { Verhallen AM, Kooistra } \\
\text { MP, van Jaarsveld BC, } \\
2007, \text { Holanda }{ }^{45} \\
\text { JCR = Q1 } \\
\text { SJR = Q1 }\end{array}$ & Estudio prospectivo & $33^{*}$ & $\begin{array}{l}\text { La técnica del ojal es útil en FAVIs } \\
\text { difíciles de canalizar, pero deben } \\
\text { aumentarse las precauciones para } \\
\text { evitar la infección }\end{array}$ \\
\hline $\begin{array}{l}\text { Lok CE, Sontrop JM, } \\
\text { Faratro R, et al, 2014, } \\
\text { Canada }{ }^{46} \\
\text { SJR }=\text { Q1 }\end{array}$ & $\begin{array}{l}\text { Estudio de cohortes } \\
\text { prospectivo }\end{array}$ & $631^{*}$ & $\begin{array}{l}\text { La tasa de infecciones de la FAVI } \\
\text { es mayor con la técnica del ojal } \\
\text { que con la técnica de la escalera }\end{array}$ \\
\hline $\begin{array}{l}\text { Schwab SJ, Oliver MJ, } \\
\text { Suhocki P, et al, 2001, } \\
\text { EEUU }{ }^{47} \\
\text { JCR }=Q 1 \\
\text { SJR }=Q 1\end{array}$ & $\begin{array}{l}\text { Estudio observacional } \\
\text { prospectivo }\end{array}$ & $42^{*}$ & $\begin{array}{l}\text { La disminución mayor del } 20 \% \\
\text { cuando el flujo es menor de } 1000 \\
\mathrm{ml} / \mathrm{min} \text {, es predictivo de una } \\
\text { estenosis y una trombosis final de } \\
\text { la FAVI }\end{array}$ \\
\hline
\end{tabular}




\section{[ Aida Delgado Ramírez, et al ]}

Factores que influyen en la supervivencia de la fístula arteriovenosa interna y su relación con la técnica de punción

\begin{tabular}{|c|c|c|c|}
\hline Autor, Año, País & Tipo de estudio & Muestra & Principales Resultados \\
\hline $\begin{array}{l}\text { Bonforte G, Pogliani D, } \\
\text { Brenna S, et al, 2010, } \\
\text { Italia }^{48} \\
\text { JCR }=\text { Q1 } \\
\text { SJR }=\text { Q1 }\end{array}$ & Estudio prospectivo & $224 *$ & $\begin{array}{l}\text { "QB stress test" junto con la } \\
\text { monitorización clínica (doppler o } \\
\text { angiografía) es capaz de detectar } \\
\text { al } 100 \% \text { de los pacientes con } \\
\text { estenosis }\end{array}$ \\
\hline $\begin{array}{l}\text { Coentrão L, Turmel- } \\
\text { Rodrigues L, 2013, } \\
\text { Portugal, Francia }{ }^{49} \\
\text { JCR }=\text { Q4 } \\
\text { SJR }=\text { Q2 }\end{array}$ & Revisión bibliográfica & No se especifica & $\begin{array}{l}\text { Añadir el examen físico de la } \\
\text { FAVI a otro tipo de monitorización } \\
\text { clínica aumenta la detección del } \\
\text { riesgo de fallo de la misma }\end{array}$ \\
\hline $\begin{array}{l}\text { Leivaditis K, Panagoutsos } \\
\text { S, Roumeliotis A, et al, } \\
2014, \text { Grecia50 } \\
\text { JCR }=\text { Q3 } \\
\text { SJR }=\text { Q3 }\end{array}$ & Revisión bibliográfica & No se especifica & $\begin{array}{l}\text { La relación de la presión } \\
\text { intraacceso y la presión arterial } \\
\text { media será }>0,5 \text { cuando exista } \\
\text { estenosis. } \\
\text { QBST positivo con flujos bajos } \\
\text { se considera un mayor riesgo de } \\
\text { estenosis. } \\
\text { Un flujo menor de } 500 \text { o } 465 \mathrm{ml} / \\
\text { min se ha asociado con estenosis }\end{array}$ \\
\hline $\begin{array}{l}\text { Kim Y0, Yang CW, Yoon SA, } \\
\text { et al, 2001, Korea } \\
\text { JCR }=\text { Q2 } \\
\text { SJR }=\text { Q2 }\end{array}$ & Estudio observacional & $58 *$ & $\begin{array}{l}\text { El riesgo de fallo de la FAVI } \\
\text { aumenta en pacientes con un flujo } \\
\text { inferior a } 350 \mathrm{ml} / \mathrm{min}\end{array}$ \\
\hline $\begin{array}{l}\text { Casey ET, Murad MH, Rizvi } \\
\text { AZ, et al, 2008, EEUU } 52 \\
\text { JCR = Q4 } \\
\text { SJR = Q1 }\end{array}$ & $\begin{array}{l}\text { Revisión sistemática y meta- } \\
\text { análisis }\end{array}$ & $14 * *$ & $\begin{array}{l}\text { No se ha encontrado evidencia } \\
\text { de calidad que fundamente la } \\
\text { vigilancia de la FAVI sana pueda } \\
\text { reducir el riesgo de trombosis }\end{array}$ \\
\hline $\begin{array}{l}\text { Tonelli M, James M, Wiebe } \\
\text { N, et al, 2008, Canada } \\
\text { JCR = Q1 } \\
\text { SJR = Q1 }\end{array}$ & $\begin{array}{l}\text { Revisión sistemática y meta- } \\
\text { análisis }\end{array}$ & 12 ** & $\begin{array}{l}\text { La detección del flujo sanguíneo es } \\
\text { útil para detectar la trombosis de } \\
\text { la FAVI pero no hay evidencia de } \\
\text { que evite la pérdida del acceso }\end{array}$ \\
\hline
\end{tabular}

Muestra: ${ }^{*}=$ pacientes; ${ }^{* *}=$ artículos. JCR: Journal Citation Report. SJR: SCimago Journal \& Country Rank. Q: Cuartiles. 
Tabla 3. Calidad metodológica de los estudios revisados.

\begin{tabular}{|c|c|c|c|c|c|c|c|c|c|c|c|c|c|c|}
\hline & 1 & 2 & 3 & 4 & 5 & 6 & 7 & 8 & 9 & 10 & 11 & 12 & Total & Calidad \\
\hline Lazarides MK et al, 200718 & + & + & + & + & - & + & + & + & + & + & + & + & 11 & Alta \\
\hline Cheng Q, Zhao YJ, 201519 & + & + & - & + & + & + & + & + & + & - & - & + & 9 & Alta \\
\hline Ocak G et al, 201311 & + & + & + & + & + & + & + & + & + & + & + & + & 12 & Alta \\
\hline Chang TI et al, 201112 & - & - & + & + & + & + & + & + & + & - & + & + & 9 & Alta \\
\hline Farber A et al, 201620 & + & + & + & + & - & + & + & + & + & - & + & + & 10 & Alta \\
\hline Bashar K et al, 201521 & + & + & + & + & - & + & + & + & + & + & + & + & 11 & Alta \\
\hline Salmela B et al, 201322 & + & + & + & + & - & + & + & + & + & + & + & + & 11 & Alta \\
\hline Masengu A et al, 201623 & + & - & + & + & - & + & + & + & + & - & + & + & 9 & Alta \\
\hline Tirinescu DC et al, 201524 & + & + & + & + & - & + & + & + & + & + & - & + & 10 & Alta \\
\hline Moon J-Y et al, 201525 & + & + & - & + & - & + & + & + & + & + & + & + & 10 & Alta \\
\hline Afsar B, Elsurer R, 201226 & + & + & + & + & + & + & + & + & + & + & + & + & 12 & Alta \\
\hline Protack CD et al, 201227 & + & + & - & + & + & + & + & + & + & - & + & + & 10 & Alta \\
\hline Verschuren JJW et al, 201328 & + & + & + & + & + & + & + & + & + & - & + & + & 11 & Alta \\
\hline Memetoglu ME et al, 201529 & + & + & - & + & + & + & + & + & + & - & + & + & 10 & Alta \\
\hline Bilgic A et al, 200730 & + & + & - & + & + & + & + & - & + & - & + & + & 9 & Alta \\
\hline Hruby Z et al, 201331 & - & + & + & - & - & + & + & + & + & - & + & + & 8 & Media \\
\hline Chang C-J et al, 200532 & - & + & - & + & + & + & + & - & + & - & + & + & 8 & Media \\
\hline Brahmbhatt A et al, 201633 & - & + & - & - & - & + & + & - & + & - & + & + & 6 & Media \\
\hline Milburn JA et al, 201334 & + & + & + & + & + & + & + & + & + & - & + & + & 11 & Alta \\
\hline Bataille S et al, 201535 & - & + & + & + & + & + & + & + & - & - & + & + & 9 & Alta \\
\hline Jamshid R et al, 200336 & - & + & + & + & - & + & + & + & + & - & + & + & 9 & Alta \\
\hline Sánchez Gonzalez, JC, 201337 & + & + & + & + & - & + & + & + & + & + & + & + & 11 & Alta \\
\hline Yoo DW, Yoon M, Jun HJ, 201438 & - & + & + & - & - & + & + & + & + & - & + & + & 8 & Media \\
\hline Grandaliano G et al, 200339 & - & + & + & + & - & + & + & + & + & + & + & + & 10 & Alta \\
\hline Muir CA et al, 201440 & + & + & + & + & - & + & + & + & + & + & + & + & 11 & Alta \\
\hline Parisotto MT et al, 201441 & - & + & + & + & - & + & + & + & + & + & + & + & 10 & Alta \\
\hline Kandil H et al, 201442 & - & + & + & + & - & + & + & + & + & - & + & + & 9 & Alta \\
\hline MacRae JM et al, 201243 & + & + & + & + & - & + & + & + & + & + & + & + & 11 & Alta \\
\hline Béchade C et al, 201544 & - & + & + & + & - & + & + & + & + & - & + & + & 9 & Alta \\
\hline Verhallen AM et al, 200745 & + & + & - & + & - & + & + & + & + & - & + & + & 9 & Alta \\
\hline
\end{tabular}


Tabla 3. Calidad metodológica de los estudios revisados.

\begin{tabular}{|c|c|c|c|c|c|c|c|c|c|c|c|c|c|c|}
\hline & 1 & 2 & 3 & 4 & 5 & 6 & 7 & 8 & 9 & 10 & 11 & 12 & Total & Calidad \\
\hline Lok CE et al, 201446 & - & + & + & + & + & + & + & + & + & - & + & + & 10 & Alta \\
\hline Schwab SJ et al, 200147 & - & + & + & + & - & + & + & + & - & - & + & + & 8 & Media \\
\hline Bonforte G et al, 201048 & + & + & + & + & - & + & + & + & + & + & + & + & 11 & Alta \\
\hline Coentrão L, Turmel-Rodrigues L, 201349 & - & + & - & - & - & + & + & - & - & - & + & - & 4 & Baja \\
\hline Leivaditis $\mathrm{K}$ et al, 201450 & - & - & - & - & - & + & + & - & - & - & + & + & 4 & Baja \\
\hline Kim Y0 et al, 200151 & - & + & - & + & - & + & + & + & - & + & + & + & 8 & Media \\
\hline Casey ET et al, 200852 & + & + & + & + & - & + & + & + & + & + & + & + & 11 & Alta \\
\hline Tonelli M et al, 200853 & + & + & + & + & - & + & + & + & + & + & + & + & 11 & Alta \\
\hline
\end{tabular}

Criterios adaptados de Berra, Elorza-Ricart, Estrada y Sánchez, 2008.

Selección y diseño de investigación: 1 . Se indican los criterios de inclusión y/o exclusión de los pacientes; 2 . Se especifica el método de selección de la muestra; 3 . Se especifica el diseño de investigación claramente en el texto; 4 . Se informa del número de pacientes potencialmente elegibles y/o los inicialmente seleccionados y/o los que aceptan y/o las que finalmente participan o responden; 5 . Si se comparan grupos, se indica la información del punto 4 para cada grupo.

Definición y medición de las variables estudiadas: 6. Se definen claramente las variables estudiadas; 7 . Se utilizan instrumentos validados para todas las variables principales estudiadas.

Método y análisis de datos: 8. Las muestras evaluadas son iguales o mayores a 30 pacientes; 9 . Se especifican las pruebas estadísticas utilizadas; 10 . Se trataron correctamente la pérdida de participantes y/o datos perdidos (o al menos está indicada en el texto que se ha revisado la calidad de los datos antes del análisis estadístico).

Calidad de los resultados y discusión: 11. Los resultados están claramente descritos de acuerdo con los objetivos del estudio; 12. La discusión considera implicaciones prácticas de los resultados y potenciales beneficios para los pacientes.

Calidad: 1-4 puntos: baja; 5-8 puntos: media; 9-12 puntos: alta.

\section{Análisis de los resultados en función de las variables}

\section{Edad}

Según diversos estudios ${ }^{18,19}$ uno de los factores de riesgo para que se produzca el fallo de la FAVI, es la edad avanzada, debido a que con el paso del tiempo las paredes de los vasos pierden elasticidad y se endurecen, a lo que se suma que en las personas de edad avanzada son frecuentes enfermedades colaterales a la ERCA como diabetes, hipertensión arterial, o cardiopatías, lo cual también puede influir en la dinámica vascular.

Esto es especialmente relevante en el metaanálisis ${ }^{18}$, en el que se encuentra evidencia de que la edad avanzada incrementa en un $50 \%$ el riesgo de fallo primario de la FAVI, y una odds ratio de 1.357 para el fallo secundario, en estos pacientes.

\section{Género}

En diferentes estudios se ha asociado el fallo de la FAVI, tanto primario como secundario, al género femenino ${ }^{20-23}$. Por tanto, ser mujer es un factor de riesgo independiente, debido a que generalmente, las mujeres tienen un diámetro vascular más pequeño que los hombres y también se atribuye al mayor tejido adiposo subcutaneo ${ }^{22,23}$. Aunque las causas no están del todo establecidas, las diferencias en el fallo de la FAVI entre hombres y mujeres son estadísticamente significativas.

Sin embargo, en un estudio ${ }^{24}$ se ha comunicado to contrario, es decir, que se produce más estenosis y posterior trombosis de la FAVI en el género masculino, aunque se debe tener en cuenta que el tamaño de la muestra $(n=97)$ es mucho más pequeño en comparación a los otros estudios. 


\section{Factores metabólicos}

Otros factores a los que se atribuyen habitualmente el fallo de la FAVI, son los metabólicos, como son la diabetes, la hipertensión o el síndrome metabólico 19,25-27; aunque se ha observado también asociación con factores del metabolismo mineral relacionados con la calcificación de los vasos ${ }^{19,24,25}$.

Varios estudios ${ }^{19,25,26}$, han mostrado que la diabetes, como factor de riesgo independiente, está íntimamente relacionada con la perdida de permeabilidad de la FAVI lo que se traduce en el fallo, tanto primario como secundario, de la misma. Aunque uno de estos estudios ${ }^{26}$, ha señalado que son los diabéticos con un mal control de la glucemia, y una hemoglobina glicosilada mayor de $7 \%$, los que tienen mayor riesgo de fallo de la FAVI.

Además, la diabetes asociada a otros trastornos como la hipertensión o la dislipemia ${ }^{19}$, y el síndrome metabólico ${ }^{27}$, contribuyen a la disfunción de la FAVI ya que conllevan cambios vasculares: pueden dañar la íntima debido a procesos proinflamatorios y protrombóticos, y disminuir el flujo sanguíneo.

En cuanto a la calcificación de los vasos, se ha asociado con niveles altos de hormona paratiroidea, y desórdenes en la regulación del metabolismo del fósforo y el calcio; esta calcificación provoca dificultades en la maduración de la FAVI, lo que se traduce en una pérdida de permeabilidad temprana con la consiguiente estenosis y posterior fracaso de la misma ${ }^{19,24,25}$.

\section{Estado inflamatorio y de mecanismos de la coagulación}

El estado inflamatorio persistente, que está íntimamente relacionado con un estado de hipercoagulabilidad, ha sido identificado como factor de riesgo de trombosis de la FAVI'1.

Varios estudios ${ }^{11,28-33}$ han encontrado que diferentes marcadores de la inflamación, se han visto relacionados con el fallo de la FAVI: niveles altos de Proteína $C$ reactiva $(P C R)^{11,29,30}$ y fetuina- $A^{12}$, presencia de factor $V$ de Leiden y gen LRPI rsl46653528, presencia de interleucina-6 $6^{30,32}$ e inhibidor del activador del plasminógeno- ${ }^{29}$, actividad de los factores de crecimiento de la pared vascular PDGFß1 y TGFß31, aumento de la expresión de VCAM-1 y citocinas proinflamatorias ${ }^{32}$.
Con respecto al estado de hipercoagulabilidad, la trombofilia ${ }^{22,28}$ es uno de los factores que con más frecuencia se relacionan con el fallo de la FAVI. También se consideran como marcadores de este estado, el receptor endotelial de la proteína $\mathrm{C}^{30}$, niveles altos del complejo trombina-antitrombina ${ }^{34}$, y la presencia de anticuerpos antifosfolípidos, especialmente el anticoagulante lúpico ${ }^{35}$.

\section{Hipotensión}

Los episodios repetidos de hipotensión intradiálisis y niveles bajos de presión arterial pre y postdiálisis, se han establecido como factores de riesgo para el fallo de la FAVI'12.

Ambas situaciones, pueden ocasionar situaciones hemodinámicas extremas, debido a la consiguiente vasoconstricción y la disminución del flujo sanguíneo en las extremidades, que afecten de forma negativa al acceso, siendo predictores de trombosis de forma independiente ${ }^{12}$.

Un estudio ha destacado, que episodios de hipotensión cercanos al acto quirúrgico del AV, favorecen la formación de trombos, ya que disminuyen el flujo sanguíneo, y por tanto, existe un mayor tiempo de contacto entre plaquetas, factores de coagulación y las células endoteliales ${ }^{19}$.

\section{Localización distal FAVI}

Existe abundante bibliografía $18,20,23,24,36$, que ha concluido que una localización distal para la creación del acceso vascular está directamente relacionada con el fallo del mismo, tanto primario como secundario.

A pesar, de que se suele preferir la construcción de la FAVI en una zona distal de miembros superiores, generalmente radio-cefálica, para preservar zonas más proximales y utilizarlas en caso de fallo de la primera $^{18}$, las FAVI radio-cefálicas presentan mayor tasa de fallo en la maduración que las braquio-cefálicas o braquio-basílicas ${ }^{18,20,24,36}$.

Esto puede ser debido a que el calibre arterial está inversamente relacionado con la tasa de trombosis $^{19,20}$, por tanto, la arteria braquial al ser de mayor calibre que la arteria radial, provoca menos incidencia de trombosis, aunque mayor incidencia del síndrome de "robo arterial". 


\section{Uso previo CVC}

Se ha relacionado el ser portador de un CVC, previamente a la creación de la FAVI, con la trombosis de la misma 24,37,38; incrementándose a casi el doble el riesgo de sufrir trombosis del acceso en uno de estos estudios ${ }^{37}$.

El hecho de ser portador de un CVC conlleva numerosos riesgos, como tasas más altas de infección y estenosis profunda de la vena subclavia ${ }^{38}$, lo que puede también influir en provocar el fracaso de la FAVI.

\section{Tratamiento con EPO}

La influencia en la patogénesis de la trombosis de la FAVI del tratamiento con eritropoyetina (EPO) ha sido muy controvertido. Algunos estudios han comunicado que aquellos pacientes a los que se les administra EPO tienen tasas más altas de fallo o trombosis de la $\mathrm{FAVI}^{34,39}$, asociándose significativamente la dosis semanal con el fracaso de la FAVI. Se ha observado que a dosis más altas, el riesgo aumenta, debido a que se induce un mayor estado inflamatorio ${ }^{39}$, presentando un aumento de los receptores de la proteína C, como ya se ha comentado anteriormente; por lo que el tratamiento con EPO podría considerarse un factor de riesgo independiente.

Por otro lado, se ha observado que en aquellas FAVIs en las que se ha producido una estenosis, existe un aumento significativo de los receptores de la EP0, lo cual puede favorecer la trombosis ${ }^{34}$.

\section{Técnica de punción}

La técnica de punción es un factor que merece ser tenido en cuenta en la supervivencia de la FAVI ${ }^{40-46}$, puesto que se está manipulando directamente el acceso, y además es casi exclusivamente responsabilidad de enfermería; por tanto, se debe ser consecuente y responsable con respecto a la técnica que se lleve a cabo, pues la punción siempre debe realizarse de forma aséptica y meticulosa, habiendo valorado previamente la vena arterializada del paciente, evitando siempre zonas enrojecidas o con supuración, zonas de hematoma, costra y/o piel alterada y zonas de aneurismas o pseudo-aneurismas ${ }^{54}$.

Existen tres tipos de técnicas para canalizar la FAVI: la técnica en escalera, la punción por zonas o área, y la técnica del ojal ${ }^{41}$. La mayor parte de la bibliografía se centra en los pros y los contras de la técnica del ojal ${ }^{40,42-46}$, cuya utilización se está incrementando de forma considerable en los últimos años y sobre la que existe una mayor controversia.

Casi todos los estudios encontrados han señalado que la técnica del ojal es la que reporta mayores tasas de infección con respecto a las otras técnicas, y mayor dificultad para realizar la hemostasia al finalizar la sesión de diálisis, debido a que se manipula constantemente el mismo orificio de entrada al acceso $00,42,43,45,46$. Por otro lado, también hay autores que defienden esta técnica, afirmando que la tasa de infección de nueva aparición no aumenta44 o que es mayor con la técnica de punción por zonas, con esta última las complicaciones se centran en que es mayor el riesgo de provocar un aneurisma o pseudo-aneurisma ${ }^{41}$.

A pesar de la evidencia en contra de la técnica del ojal, también se ha sugerido que es la técnica ideal para fístulas "difíciles" de canalizar o con trayecto corto, y en HD domiciliaria o en centros donde la rotación del personal de enfermería sea mínima, ya que se punciona siempre en el mismo sitio y con la misma inclinación, por lo que hay menor probabilidad de fa$110^{42,45,46,55}$. En España se ha llevado a cabo una investigación sobre el uso de esta técnica, mostrando que a pesar de ser conocida en la mayoría de centros de diálisis, no se suele llevar a la práctica, principalmente porque su conocimiento es eminentemente teórico y no muy profundo, y por tanto, los enfermeros no suelen atreverse a utilizarla porque no están formados al respecto ${ }^{56}$.

Con respecto a la punción con cualquiera de las técnicas, se ha comunicado que existe una menor supervivencia de la FAVI, cuando se realiza la punción con la aguja arterial en dirección retrógada, colocando el bisel hacia abajo, aplicando torniquete o no haciendo la presión correcta para realizar la hemostasia ${ }^{41}$. A este respecto, hay que indicar que la asociación "punción con la aguja arterial en dirección retrógada, colocando el bisel hacia abajo" como variable para analizar la supervivencia de la FAVI puede haber incurrido en un sesgo de selección, pues al tratarse de estudio observacional, sin intervención, cabe la posibilidad que a los pacientes que se les puncionaba de esta forma tuvieran previamente un déficit de flujo intraacceso, que es excelente predictor de trombosis en las FAVIs ${ }^{51,52}$.

Otro dato a tener en cuenta, es que una tasa de eventos infecciosos más alta puede inducir un mayor esta- 
do inflamatorio ${ }^{43}$, que como ya se ha analizado anteriormente, puede influir en la aparición de trombosis del acceso $^{40}$. En cualquier caso no es posible esclarecer cuál es la mejor técnica de punción para aumentar la supervivencia de la FAVI, puesto que las ventajas e inconvenientes de cada una dependen mucho del paciente y de las características del AV que tenga.

\section{Monitorización/Vigilancia}

Se ha documentado ampliamente que las técnicas de monitorización y vigilancia de la FAVI son útiles para detectar la estenosis ${ }^{47-53}$. Esto es muy importante pues en un porcentaje muy alto la trombosis de la FAVI va precedida de estenosis ${ }^{9}$. En la mayoría de los estudios revisados han utilizado la medición de flujo intraacceso mediante ecografía doppler o dilución por ultrasonidos $^{51,53}$, angiografía ${ }^{52}$, o el "QB stress test" (QBST) ${ }^{48}$ con las cuales se obtienen altos índices de detección de estenosis.

Con respecto a la función de enfermería en la vigilancia de la FAVI, existen técnicas útiles sin grandes costes y de fácil realización como son:

- El examen físico que consiste en inspección del tracto venoso observando cualquier cambio reseñable, palpación del pulso y el "thrill" a lo largo del acceso, y de cualquier aumento de presión, y auscultación valorando la existencia de soplos discontinuos o débiles ${ }^{49,54}$.

- La monitorización de la recirculación sanguínea, que informa del porcentaje de sangre ya depurada que vuelve nuevamente al circuito extracorpóreo, y que debe mantenerse al $10 \%$, pues una recirculación alta es indicativa de flujos intraacceso bajos, lo que puede hacer sospechar una estenosis ${ }^{50}$.

- La llamada "presión pre-bomba", o caída de la presión en la línea arterial, lo cual indica una disminución del flujo de la FAVI, que es predictivo de estenosis. Para medir este parámetro se deben tener en cuenta, el calibre de la aguja y su posición, y el flujo fijado en el monitor de la diálisis ${ }^{10}$.

- La medición del flujo intraacceso, utilizando la técnica de dilución por ultrasonidos, de la misma forma que la anterior, una disminución mayor del $20 \%$ cuando el flujo es menor de $1000 \mathrm{ml} / \mathrm{min}$, es predictivo de una estenosis que puede conducir a una trombosis final del acceso $0^{47}$. Otro método validado para medir el flujo intraacceso es mediante termodilución con monitor de temperatura sanguínea $\left(\mathrm{BTM}^{\circledR}\right)$, cuyos resultados tienen muy buena correlación con el método de dilución por ultrasonidos y no requiere monitores anexos al monitor de hemodiálisis ${ }^{57}$. También se ha documentado riesgo de estenosis con QBST positivo y flujos bajos, entendiéndose por bajos aquellos menores de 500 o $465 \mathrm{ml} / \mathrm{min}^{50}$.

Sin embargo, a pesar de que está demostrado que la vigilancia es útil para detectar la estenosis, hay bibliografía que afirma, que al margen de que se lleve a cabo la detección de la misma, no es igualmente útil para prevenir la trombosis y la posterior pérdida del acces $0^{52-53}$, puesto que una vez producida la estenosis es difícil frenar la evolución al fracaso de la FAVI.

\section{Consideraciones prácticas}

Como se ha puesto de manifiesto, la detección de la estenosis es fundamental como prevención de la trombosis o fallo de la FAVI, mediante técnicas de monitorización y vigilancia, con procedimientos complejos como la ecografía doppler, la angiografía, el QBST o la medición del flujo intraacceso, o procedimientos más sencillos y asequibles para enfermería como el examen físico, la monitorización de la recirculación, o de la caída de la presión pre-bomba y la presión venosa.

Con respecto a la utilización de las diferentes técnicas de punción de la FAVI, no hay datos concluyentes a favor de la utilización de ninguna de las 3 técnicas, pues siempre hay que individualizar según tipo de $\mathrm{AV}$; si bien la técnica del ojal se ha relacionado con mayor tasa de infecciones. Por ello, esta técnica se recomienda para pacientes en HD domiciliaria por la guías KD0QI58 o en unidades con poca rotación de enfermeras por paciente.

Como recomendación general, es importante realizar la punción de forma aséptica y siempre que ello sea posible, utilizar la técnica en escalera o rotación de los puntos de punción, que además es obligatorio en el caso de los injertos de PTFE. 


\section{Conclusiones}

Según los resultados de esta revisión, las variables que más influyen en una peor supervivencia de la FAVI son las siguientes:

- Edad avanzada y género femenino.

- Causas metabólicas, especialmente la diabetes.

- Estado inflamatorio e hipercoagulabilidad, actuando como factores conjuntos.

- Situaciones de hipotensión en periodo previo y posterior a la sesión de HD, y cuando la hipotensión se produce próxima al acto quirúrgico de realización de la fístula en el fallo primario.

- Localización distal (FAVI radio-cefálicas) por pequeño calibre de la arteria radial.

- Haber sido portador de CVC, previamente a la realización de la fístula.

- En cuanto a la influencia de las diferentes técnicas de punción utilizadas en la FAVI, en la supervivencia de la misma, no hay datos concluyentes a favor de la ninguna de las 3 técnicas.

Por último decir, que desde el punto de vista de enfermería es fundamental incluir en el cuidado y manejo de la FAVI la detección de los factores de riesgo modificables, extremar las precauciones en aquellos no modificables, y hacer partícipe, mediante educación sanitaria al paciente del autocuidado de su AV.

Recibido: 25 junio 16

Revisado: 30 junio 16

Modificado: 5 julio 16

Aceptado: 1 agosto 16

\section{Bibliografía}

1. Lorenzo V, López Gómez JM. Enfermedad Renal Crónica. [Grupo Editorial]. Nefrología al Día. [consultado el 23 de marzo de 2016] D0I:10.3265/Nefrologia.2010.publ.ed80.chapter2802.

2. MSSSI. Documento Marco sobre Enfermedad Renal Crónica(ERC) dentro de la Estrategia de Abordaje a la Cronicidad en el SNS. $1^{\text {a }}$ ed. España: MSSI; Febrero 2015.

3. Registro de la Sociedad Española de Nefrología [Internet]. [Consultado el 23 de marzo de 2016]. Disponible en: http://www.senefro.org/contents/ webstructure/REER_Cong_SEN_2015.pdf.

4. Datos Históricos Donación y Trasplante España [Internet]. [consultado el 25 de marzo de 2016]. Disponible en: https://reports.ont.es/datoshistoricos.aspx.

5. ERA-EDTA Registry [Internet]. [Consultado el 29 de marzo de 2016] Disponible en: https:// www.era-edta-reg.org/files/annualreports/pdf/AnnRep2013.pdf.

6. Siddiky A, Sarwar K, Ahmad N, Gilbert J. Management of arteriovenous fistulas. BMJ. 30 de octubre de 2014;349:g6262. D0I:10.1136/bmj.g6262.

7. Roca Tey R. El acceso vascular para hemodiálisis: la asignatura pendiente. Nefrología (Madrid). 2010;30(3):280-7. DOI:10.3265/Nefrologia. pre2010.Apr.10349.

8. Martín, I. J., Minguela, J. I., Ocharan-Corcuera, J., Chena, A., \& Ruiz-de-Gauna, R. Indicaciones y tipos de accesos para Hemodiálisis. Diálisis y Trasplante; 2008. 29(4), 173-176.

9. Van Tricht I, De Wachter D, Tordoir J, Verdonck P. Hemodynamics and complications encountered with arteriovenous fistulas and grafts as vascular access for hemodialysis: a review. Ann Biomed Eng. septiembre de 2005;33(9):1142-57. DOI:10.1007/s10439-005-5367-X.

10. Sociedad Española de Nefrología. Guías de Acceso Vascular en Hemodiálisis [Internet]. [consultado el 30 de marzo de 2016]. Disponible en: http:// www.senefro.org/modules/webstructure/files/ guia_acceso_vascular.pdf?check_idfile=984.

11. Ocak G, Rotmans JI, Vossen CY, Rosendaal FR, Krediet RT, Boeschoten EW, et al. Type of arteriovenous vascular access and association with patency and mortality. BMC Nephrol. 2013;14:79. D0I:10.1186/1471-2369-14-79.

12. Chang TI, Paik J, Greene T, Desai M, Bech F, Cheung $A K$, et al. Intradialytic hypotension and vascular access thrombosis. J Am Soc Nephrol. agosto de 2011;22(8):1526-33. DOI: 10.1681/ ASN.2010101119. 
13. De-La-Fuente, Natalia, et al. "Complicaciones no trombóticas en los accesos vasculares para hemodiálisis." Diálisis y Trasplante 29.4 (2008): 214-220.

14. Barba Vélez A; Ocharan Corcuera J, Foraster A. Manejo de los accesos vasculares para hemodiálisis. Gaceta Médica de Bilbao. 2011;108(4):108113. D0I:10.1016/j.gmb.2011.09.001.

15. Victoria Miranda-Camarero M. Cuidados de las fístulas arteriovenosas. Intervenciones y actividades del profesional de enfermería. Diálisis y Trasplante. enero de 2010;31(1):12-6.

16. Vachharajani TJ. How is Arteriovenous Fistula Longevity Best Prolonged?: The Role of Cannulation and Fistula Care. Asif $A$, editor. Seminars in Dialysis. enero de 2015;28(1):24-7. D0I:10.1111/ sdi.12306.

17. Barra S, Elorza-Ricart JM, Sánchez E. Instrumento para la lectura crítica y la evaluación de estudios epidemiológicos transversales. Gac Sanit 2008;22(5):492-7.

18. Lazarides MK, Georgiadis GS, Antoniou GA, Staramos DN. A meta-analysis of dialysis access outcome in elderly patients. J Vasc Surg. febrero de 2007;45(2):420-6. DOI :10.1016/j. jvs.2006.10.035.

19. Cheng $Q$, Zhao YJ. The reasons for the failure of the primary arteriovenous fistula surgery in patients with end-stage renal disease. J Vasc Access. noviembre de 2015;16Suppl 10:S74-7. D0I:10.5301/jva.5000424.

20. Farber A, Imrey PB, Huber TS, Kaufman JM, Kraiss LW, Larive B, et al. Multiple preoperative and intraoperative factors predict early fistula thrombosis in the Hemodialysis Fistula Maturation Study. J Vasc Surg. enero de 2016;63(1):163-70.e6.D0I:http://dx.doi.org/10.1016/j.jvs.2015.07.086.

21. Bashar K, Zafar A, Elsheikh S, Healy DA, Clarke-Moloney $M$, Casserly $L$, et al. Predictive parameters of arteriovenous fistula functional maturation in a population of patients with end-stage renal disease. PLoS ONE. 2015;10(3):e0119958. DOI:10.1371/journal. pone.0119958.
22. Salmela B, Hartman J, Peltonen S, Albäck A, Lassila R. Thrombophilia and arteriovenous fistula survival in ESRD. Clin J Am Soc Nephrol. junio de 2013;8(6):962-8. D0I: 10.2215/CJN.03860412.

23. Masengu $A$, Maxwell AP, Hanko JB. Investigating clinical predictors of arteriovenous fistula functional patency in a European cohort. Clin Kidney J. febrero de 2016;9(1):142-7. D0I:10.1093/ckj/ sfv131.

24. Tirinescu DC, Bondor CI, Kacso IM. Hierarchy of risk factors for stenosis of arteriovenous fistula in chronic hemodialysis patients using TOPSIS method. Hum Vet Med. 2015;7(4):283-289.

25. Moon J-Y, Lee HM, Lee S-H, Lee T-W, Ihm C-G, Jo $\mathrm{Y}-\mathrm{I}$, et al. Hyperphosphatemia is associated with patency loss of arteriovenous fistula after 1 year of hemodialysis. Kidney Res Clin Pract. marzo de 2015;34(1):41-6.D0I:http://dx.doi.org/10.1016/j.krcp.2015.02.001.

26. Afsar B, Elsurer R. The primary arteriovenous fistula failure-a comparison between diabetic and non-diabetic patients: glycemic control matters. Int Urol Nephrol. abril de 2012;44(2):575-81. D0I:10.1007/s11255-011-9978-x.

27. Protack $C D$, Jain $A$, Vasilas $P$, Dardik A. Theinfluence of metabolic syndrome on hemodialysis access patency. J Vasc Surg. diciembre de 2012;56(6):1656-62. DOI:10.1016/j. jvs.2012.05.104.

28. Verschuren JJW, Ocak G, Dekker FW, Rabelink TJ, Jukema JW, Rotmans JI. Candidate gene analysis of arteriovenous fistula failure in hemodialysis patients. Clin J Am Soc Nephrol. agosto de 2013;8(8):1358-66. DOI: 10.2215/ CJN.11091012.

29. Memetoglu ME, Kehlibar T, Yilmaz M, Kocaaslan C, Günay R, Arslan IY, et al. Red blood cell distribution width is associated with early failure of arteriovenous fistula for haemodialysis access. Blood Coagul Fibrinolysis. enero de 2015;26(1):32-5. D0I:10.1097/MBC.0000000000000142.

30. Bilgic A, Ozdemir FN, Bayraktar N, Karakus S, Sasak G, Arat Z, et al. Soluble endothelial protein $C$ receptor: influence on arteriovenous fistula thrombosis development in hemodialysis 
patients. Am J Nephrol. 2007;27(4):366-72. D0I:10.1159/000103911.

31. Hruby Z, Stanek-Piotrowska M, Turek J, Witkiewicz W, Jonkisz A, Konieczny A, et al. The clinicopathological determinants of native arteriovenous fistula failure in patients on maintenance hemodialysis. Adv Clin Exp Med. agosto de 2013;22(4):495-500.

32. Chang C-J, Ko Y-S, Ko P-J, Hsu L-A, Chen C-F, Yang C-W, et al. Thrombosed arteriovenous fistula for hemodialysis access is characterized by a marked inflammatory activity. Kidney Int. septiembre de 2005;68(3):1312-9.

33. Brahmbhatt A, Remuzzi A, Franzoni M, Misra $S$. The molecular mechanisms of hemodialysis vascular access failure. Kidney Int. febrero de 2016;89(2):303-16.D0I:http://dx.doi. org/10.1016/j.kint.2015.12.019.

34. Milburn JA, Ford I, Mutch NJ, Fluck N, Brittenden J. Thrombin-anti-thrombin levels and patency of arterio-venous fistula in patients undergoing haemodialysis compared to healthy volunteers: a prospective analysis. PLoS ONE. 2013;8(7):e67799. D0I:10.1371/journal.pone.0067799.

35. Bataille $S$, Burtey $S$, Decourt $A$, Frère $C$, Henneuse A, Aillaud M-F, et al. [Antiphospholipids antibodies and hemodialysis: a frequent association linked to arteriovenous fistula thrombosis]. Nephrol Ther. febrero de 2015;11(1):27-33.D0I:http://dx.doi. org/10.1016/j.nephro.2014.08.005.

36. Jamshid R, Reza SA, Abbas G, Raha A. Incidence of arteriovenous thrombosis and the role of anticardiolipin antibodies in hemodialysis patients. Int Urol Nephrol. 2003;35(2):275-82.

37. Sánchez González, JC. Factores de riesgo asociados a la disfunción de una fístula arteriovenosa en pacientes con enfermedad renal crónica. Enfermería Nefrológica. 2013; 16 (2):104-114.

38. Yoo DW, Yoon M, Jun HJ. Successful Access Rate and Risk Factor of Vascular Access Surgery in Arm for Dialysis. Vasc Specialist Int. marzo de 2014;30(1):33-7.D0I:http://dx.doi.org/10.5758/ vsi.2014.30.1.33.

39. Grandaliano G, Teutonico A, Allegretti A, Losappio $R$, Mancini $A$, Gesualdo $L$, et al. The role of hyperparathyroidism, erythropoietin therapy, and CMV infection in the failure of arteriovenous fistula in hemodialysis. Kidney Int. agosto de 2003;64(2):715-9.

40. Muir CA, Kotwal SS, Hawley CM, Polkinghorne $K$, Gallagher MP, Snelling $P$, et al. Buttonhole cannulation and clinical outcomes in a home hemodialysis cohort and systematic review. Clin J Am Soc Nephrol. enero de 2014;9(1):110-9. D0I:10.2215/CJN.03930413.

41. Parisotto MT, Schoder VU, Miriunis C, Grassmann AH, Scatizzi LP, Kaufmann P, et al. Cannulation technique influences arteriovenous fistula and graf tsurvival. Kidney Int. octubre de 2014;86(4):7907. DOI:10.1038/ki.2014.96.

42. Kandil H, Collier S, Yewetu E, Cross J, Davenport A. Arteriovenous fistula survival with buttonhole (constant site) cannulation for hemodialysis access. ASAIO J. febrero de 2014;60(1):95-8. D0I:10.1097/MAT.0000000000000018.

43. MacRae JM, Ahmed SB, Atkar R, Hemmelgarn $B R$. A randomized trial comparing buttonhole with rope ladder needling in conventional hemodialysis patients. Clin J Am Soc Nephrol. octubre de 2012;7(10):1632-8. D0I:10.2215/ CJN.02730312.

44. Béchade $C$, Goovaerts T, Cougnet $P$, Labriola L, Jadoul M, Goffin E. Buttonhole Cannulation Is Not Associated with More AVF Infections in a Low-Care Satellite Dialysis Unit: A Long-Term Longitudinal Study. PLoS ONE. 2015;10(11):e0142256. DOI:10.1371/journal.pone.0142256.

45. Verhallen AM, Kooistra MP, van Jaarsveld BC. Cannulating in haemodialysis: rope-ladder or buttonhole technique? Nephrol Dial Transplant. septiembre de 2007;22(9):2601-4. D0I:10.1093/ndt/ gfm043.

46. Lok CE, Sontrop JM, Faratro R, Chan CT, Zimmerman DL. Frequent hemodialysis fistula infectious complications. Nephron Extra. septiembre de 2014;4(3):159-67. D0I:10.1159/000366477.

47. Schwab SJ, Oliver MJ, Suhocki P, McCann R. Hemodialysis arteriovenous access: detection of stenosis and response to treatment by vascular access blood flow. Kidney Int. enero de 2001;59(1):358-62. 
48. Bonforte $G$, Pogliani $D$, Brenna $S$, Martinelli $D$, Bernardi $L E$, $D^{\prime}$ Amico $M$, et al. Validation of $Q B$ stress test as a useful tool in the detection of native arteriovenous fistula stenosis: results after 22 months of follow-up. Nephrol Dial Transplant. junio de 2010;25(6):1943-9.

49. Coentrão L, Turmel-Rodrigues L. Monitoring dialysis arteriovenous fistulae: it's in our hands. J Vasc Access. septiembre de 2013;14(3):209-15. D0I:10.5301/jva.5000141.

50. Leivaditis K, Panagoutsos S, Roumeliotis A, Liakopoulos V, Vargemezis V. Vascular access for hemodialysis: postoperative evaluation and function monitoring. Int Urol Nephrol. febrero de 2014;46(2):403-9. DOI:10.1007/s11255-013-0564-2.

51. Kim Y0, Yang CW, Yoon SA, Chun KA, Kim NI, Park JS, et al. Access blood flow as a predictor of early failures of native arteriovenous fistulas in hemodialysis patients. Am J Nephrol. junio de 2001;21(3):221-5.

52. Casey ET, Murad MH, Rizvi AZ, Sidawy AN, McGrath MM, Elamin MB, et al. Surveillance of arteriovenous hemodialysis access: a systematic review and meta-analysis. J Vasc Surg. noviembre de 2008;48(5 Suppl):48S - 54S. D0I:10.1016/j. jvs.2008.08.043.

53. Tonelli $M$, James $M$, Wiebe $N$, Jindal $K$, Hemmelgarn B, Alberta KidneyDisease Network. Ultrasound monitoring to detect access stenosis in hemodialysis patients: a systematic review. Am J Kidney Dis. abril de 2008;51(4):630-40. D0I:10.1053/j.ajkd.2007.11.025.
54. Galera Fernández A, Martínez De Merlo MT, Ochando García A. Accesos vasculares para hemodiálisis: cuidados de enfermería. Angiología. 2005;57(2):159-68.

55. Jennings WC, Galt SW, Shenoy S, Wang S, Ladenheim ED, Glickman $M H$, et al. The Venous Window Needle Guide, a hemodialysis cannulation device for salvage of uncannulatable arteriovenous fistulas. J Vasc Surg. octubre de 2014;60(4):1024-32.D0I:http://dx.doi.org/10.1016/j.jvs.2014.04.016.

56. González Molina J, Momblanch Amoros T, Moreno Aliaga C, Sanz Escriba A, Navarro Daudén L, Martí i Monros A, et al. Análisis enfermero de la presencia de la Técnica del 0jal en las unidades de hemodiálisis españolas. Enfermería Nefrológica. junio de 2013;16(2):93-8.

57. Schneditz D, Kaufman AL, Levin N. Surveillance of Access Function by the Blood Temperature Monitor. Semin Dial. diciembre de 2003;16(6):4837. DOI:10.1046/j.1525-139X.2003.16104.x.

58. Daugirdas JT, Depner TA, Inrig J, Mehrotra R, Rocco MV, Suri RS, et al. KDOQI Clinical Practice Guideline for Hemodialysis Adequacy: 2015 Update. American Journal of Kidney Diseases. 1 de noviembre de 2015;66(5):884-930. 